subtle seizures related to sensory stimuli that otherwise would be overlooked. The differential diagnosis includes idiopathic, reflex myoclonic, startle epilepsy (Ricci S et al, 1995), and hyperekplexia with hypertonia and startle reflexes induced by nose tapping, unaccompanied by electrographic epileptic discharges (Dubowitz LMS et al, 1992; Ryan SG et al, 1992).

\title{
PROLONGED QT DURING EPILEPTIC SEIZURES
}

Researchers at Western General and Royal Hospital for Sick Children, Edinburgh, UK, measured the corrected QT interval before and during seizures in a prospective study of 39 children, 1 month-16 years of age, with various epilepsies. Of a total of 156 seizures 9 were generalized tonic-clonic (5 patients), 34 absences (6 patients), 12 tonic seizures ( 6 patients), 27 temporal lobe seizures ( 14 patients), 58 frontal lobe seizures ( 4 patients), and 16 subclinical seizures ( 4 patients). Corrected QT during total seizure data compared to total pre-seizure values showed a statistically significant difference $(\mathrm{p}<0.001)$. Bazett's formula used to compare QT values found that 21 seizures in 9 patients transiently increased their corrected QT beyond normal limits, with a maximum corrected QT of $512 \mathrm{~ms}$ during a right temporal lobe seizure. Significant lengthening of corrected QT cardiac repolarization time during some epileptic seizures may have a role in sudden unexplained death in epilepsy (SUDEP). (Brotherstone R, Blackhall B, McLellan A. Lengthening of corrected QT during epileptic seizures. Epilepsia Feb 2010;51(2):221-232). (Respond: Dr Ruth Brotherstone, Department of Clinical Neurophysiology, Western General Hospital, Crewe Road, Edinburgh EH4 2XU, UK. E-mail: ruth.brotherstone@luht.scot.nhs.uk).

COMMENT. Measurements of QT in individual patients may show lengthening during some seizures, as in this study, whereas mean QT values for grouped data may be normal, as reported in some previous studies. No evidence of QT prolongation was detected in patients receiving antiepileptic monotherapy or polytherapy in a pediatric group (Kwon S et al. Pediatr Neurol 2003;30:99-101).

Cardiac arrhythmias and Seizures. In a 2-year review of electrographically confirmed seizures in a pediatric epilepsy-monitoring unit, ictal cardiac arrhythmias occurred in $45 \%$ of 244 seizures ( $40 \%$ of the patients). Benign respiratory sinus arrhythmia was the most common arrhythmia (in $78 \%$ of seizures with arrhythmias and $70 \%$ of patients with arrhythmias). Potentially serious arrhythmias including irregular variable arrhythmias and abnormal QRS intervals were seen in $12 \%$ of all the patients. (Standridge SM, Holland KD, Horn PS. Pediatr Neurol 2010;42:201-205).

Enhanced QT shortening and persistent tachycardia after generalized seizures are reported in a study of 25 patients with medically refractory temporal lobe epilepsy undergoing presurgical investigation at Institute of Neurology, Queen Square, London, UK (Surges R et al. Neurology Feb 2, 2010;74(5):421-426). Secondarily GTCS led to higher peri-ictal heart rate (HR), persistent postictal tachycardia, and decreased postictal HR variability. Abnormal shortening of the corrected QT interval occurred in 17 patients, mainly with secondarily GTCS. Benign cardiac arrhythmias occurred in 14 patients and 
were independent of seizure type. Cardiac abnormalities occurring in epilepsy with GTCS may potentially facilitate sudden cardiac death (SUDEP).

\section{ETHOSUXIMIDE, VALPROIC ACID, AND LAMOTRIGINE IN CHILDHOOD ABSENCE EPILEPSY}

Efficacy, tolerability, and neuropsychological effects of ethosuximide, valproic acid, and lamotrigine in children with newly diagnosed childhood absence epilepsy were compared in a double-blind, randomized, controlled clinical trial performed at six centers in the US and organized as a Study Group. Drug doses were incrementally increased until freedom from seizures or highest tolerable dose was reached, Primary outcome was freedom from treatment failure after 16 weeks therapy, and secondary outcome was attentional dysfunction. In a total of 453 children, the freedom-from-failure rates after 16 weeks were similar for ethosuximide $(53 \%)$ and valproic acid $(58 \%)$, and higher than the rate for lamotrigine $(29 \%)(\mathrm{P}<0.001)$. Attentional dysfunction was more common with valproic acid $(49 \%)$ than with ethosuximide $(33 \%)(\mathrm{P}=0.03)$. (Glauser TA, Cnaan A, Shinnar S, et al. Ethosuximide, valproic acid, and lamotrigine in childhood absence epilepsy. N Engl J Med March 4 2010;362:790-799). (Reprints: Dr Tracy A Glauser, Cincinnati Children's Hospital, 3333 Burnett Ave, MLC 2015, Cincinnati, OH 45229. Email: tracy.glauser@cchmc.org).

COMMENT. "Older is better," is the conclusion of Vining EPG, in an editorial (N Engl J Med 2010;362:843-845). As generally accepted in US practice and confirmed by the above controlled trial, ethosuximide from the 1950 s is the optimal initial therapy for childhood absence epilepsy without GTCS. Ethosuximide is equal to valproic acid in seizure control and superior in effects on attention. Attentiveness was significantly poorer among children receiving valproic acid than in those taking ethosuximide or lamotrigine, an important factor in the choice of long-term therapy for epilepsy in children.

\section{INEFFECTIVENESS OF ADJUNCTIVE TOPIRAMATE IN INFANTS WITH REFRACTORY PARTIAL SEIZURES}

A double-blind, placebo-controlled, international study of topiramate in 149 infants aged 1-24 months (mean age 12 months) with refractory partial-onset seizures is reported from Seattle Children's Hospital, WA. Topiramate 5, 15, or $25 \mathrm{mg} / \mathrm{kg} / \mathrm{d}$ or placebo was given for 20 days, and the percentage reduction in daily seizure rate was recorded on a 48-hour video-EEG. Infants with at least 4 seizures in the 2 weeks before the first day of screening and at least 2 electroclinical seizures in the 48 -hour baseline vEEG were eligible. The most frequently used AEDs at baseline were valproic acid (56\%), phenobarbital (29\%), and carbamazepine (17\%). The median percentage reduction from baseline in daily seizure rate was not significantly different $(\mathrm{p}=0.97)$ between topiramate $25 \mathrm{mg} / \mathrm{kg}(20.4 \%)$ and placebo (13.1\%). Comparisons of lower doses of topiramate and placebo were not significant. Similar results were obtained when the analysis was adjusted for sex, and type or number of AEDs at baseline. Treatment side effects occurred more frequently (>10\% difference) with topiramate than with placebo. These included fever, diarrhea, vomiting, anorexia, weight decrease, somnolence, and 